\title{
What is the cause of $P$-wave undersensing in this CRT-D device?
}

\author{
J. Schroemges ${ }^{1}$ F. A. L. E. Bracke' • B. M. van Gelder ${ }^{1}$
}

Published online: 26 June 2018

(c) The Author(s) 2018

In a 70-year-old male suffering from coronary artery disease in New York Heart Association class II-III with an ejection fraction of 25\%, a DDDR pacemaker was replaced by a CRT-D device. A Medtronic Claria MRI Quad was implanted in January 2017.

Recently the patient presented at the pacemaker clinic with reduced exercise tolerance and shortness of breath even after the short walk from the parking lot to the pacemaker clinic. The ECG recorded during these complaints is presented in Fig. 1 and shows $P$-wave undersensing, which normalised after a few minutes of rest. $P$-wave undersensing could easily be evoked again by the patient repeat exercising for a few minutes.
What was the cause of $P$-wave undersensing in this device?

\section{Answer}

You will find the answer elsewhere in this issue.

Open Access This article is distributed under the terms of the Creative Commons Attribution 4.0 International License (http:// creativecommons.org/licenses/by/4.0/), which permits unrestricted use, distribution, and reproduction in any medium, provided you give appropriate credit to the original author(s) and the source, provide a link to the Creative Commons license, and indicate if changes were made.
B. M. van Gelder

carlgr@cze.nl

1 Department of Electrophysiology, Catharina Hospital, Eindhoven, The Netherlands 


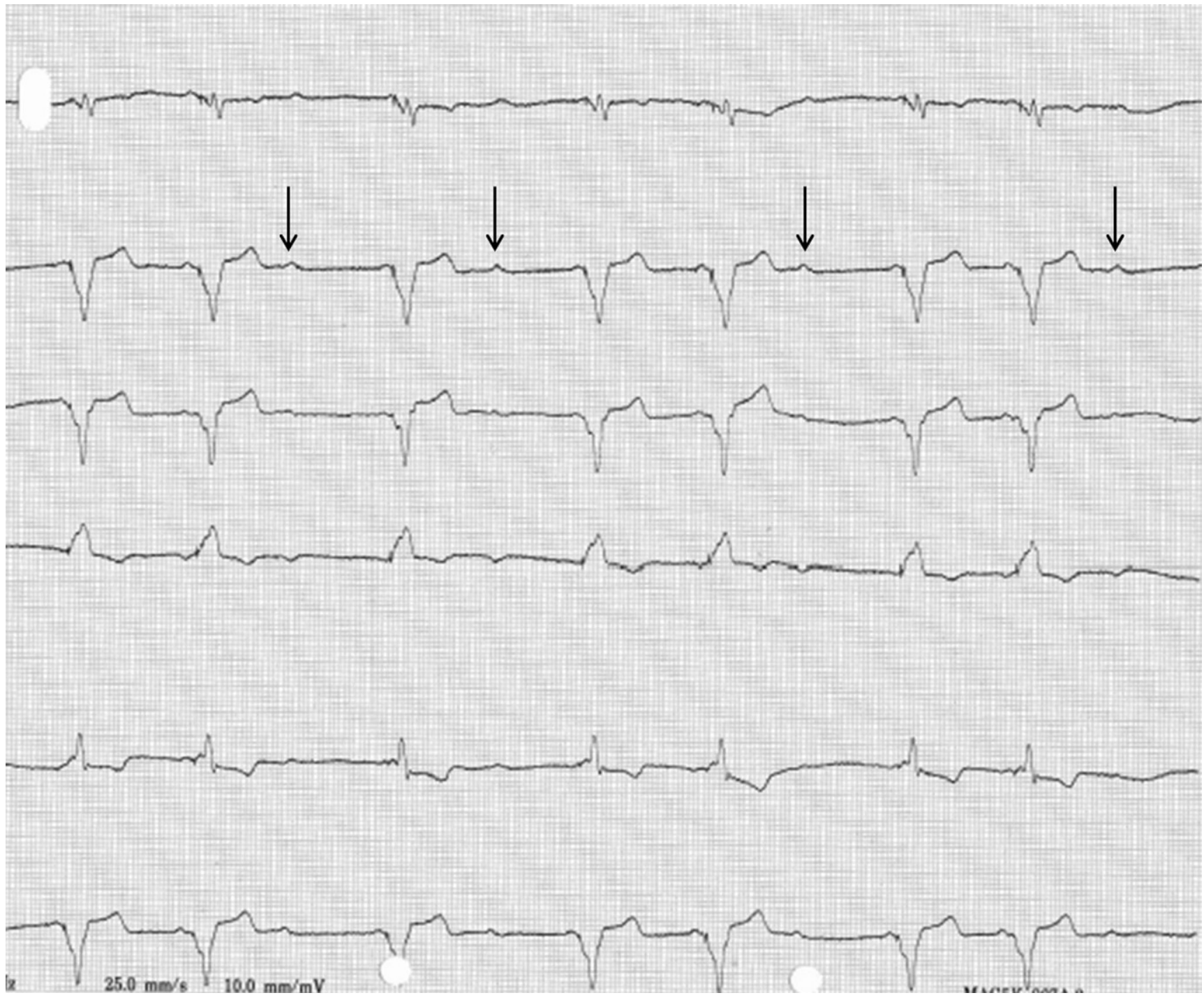

Fig. 1 From top to bottom: ECG leads I, II, III, aVR, aVL, aVF, showing atrial synchronous ventricular pacing with intermittent $P$-wave undersensing (arrows) 